\title{
Cadaver Validation of Intensity-Based Ultrasound to CT Registration
}

\author{
Graeme P. Penney ${ }^{1}$, Dean C. Barratt ${ }^{1}$, Carolyn S.K. Chan ${ }^{1}$, \\ Mike Slomczykowski ${ }^{2}$, Timothy J. Carter ${ }^{1}$, Phillip J. Edwards ${ }^{3}$, \\ and David J.Hawkes ${ }^{1}$
}

1 Centre for Medical Image Computing (CMIC), University College London, UK g.penney@cs.ucl.ac.uk

2 Department of Surgical Oncology and Technology, Imperial College London, UK 3 i-Orthopaedics, DePuy a Johnson \& Johnson Company, Leeds, UK

\begin{abstract}
A method is presented for the registration of tracked B-mode ultrasound images to a $\mathrm{CT}$ volume of a femur or pelvis. This registration can allow tracked surgical instruments to be aligned with the CT image or an associated preoperative plan. Our method requires no manual segmentation of either the ultrasound images or the CT volume. The $\mathrm{CT}$ and US images are processed to produce images where the image intensity represents the probability of the presence of a bone edge. These images are then registered together using normalised cross-correlation as a similarity measure. The parameter which represents the speed of sound through tissue has also been included in the registration optimisation process. Experiments have been carried out on six cadaveric femurs and three cadaveric pelves. Registration results were compared with a "gold standard" registration acquired using bone implanted fiducial markers. Results show the registration method to be accurate, on average, to $1.7 \mathrm{~mm}$ root-mean-square target registration error.
\end{abstract}

\section{Introduction}

Recent years have seen the emergence of image-guidance systems for orthopaedic surgery 123]. All of these systems require a registration between physical and image space. Most systems achieve this by identifying point landmarks or by delineating surfaces using a tracked pointer. To achieve accurate registrations, bony landmarks, or fiducials attached to bone should be located. Implanting fiducials or exposing additional bone surfaces for registration is invasive and may cause additional pain and risk of infection, whereas limiting surface information to regions exposed in standard procedures may adversely affect registration accuracy. There is a trade off between invasiveness and accuracy of the registration process. The drive to develop less invasive registration methods will increase with the adoption of minimally invasive surgical techniques [4.5], particularly as reduced invasiveness of procedures may be the most powerful argument for the use of computer assisted orthopaedic surgery systems. 
Proposals to use B-mode ultrasound (US) for registration to bone in image guided surgery go back approximately a decade [6]. The main application areas have involved registrations on vertebrae 78 , pelves 98010 and long bones 6 61112. The work presented in this paper differs from previously published work in three main ways: firstly, we have used an intensity-based algorithm, therefore no segmentation is required in either the US or CT volume; secondly, our algorithm also optimises the probe calibration parameter which is directly related to the average speed-of-sound through tissue; and thirdly, our validation strategy uses a combination of human cadavers, an independently calculated "gold standard" registration based on bone implanted fiducial markers, and clinically realistic starting positions.

\section{Method}

\subsection{Overview of System}

The registration algorithm used in this paper is an extension of an algorithm previously described for registering US images to magnetic resonance images of the liver 13. An overview of the registration system is given in Figure 1 An optical localiser (Optotrak 3020, Northern Digital Inc., Ontario, Canada) was used to track an US probe and a dynamic reference object (DRO) which is rigidly attached to the bone. Our aim is to calculate the registration transformation, $\mathbf{T}_{r e g}$, which transforms physical positions in the coordinate system of the DRO into voxel positions within a preoperative $\mathrm{CT}$ volume.

To calculate $\mathbf{T}_{r e g}$, 3D freehand US images are acquired of the bone surface. These images are then registered to the preoperative CT volume to obtain transformation, $\mathbf{T}$, which maps pixel positions, $\mathbf{x}_{U S}$, in the US images to voxel

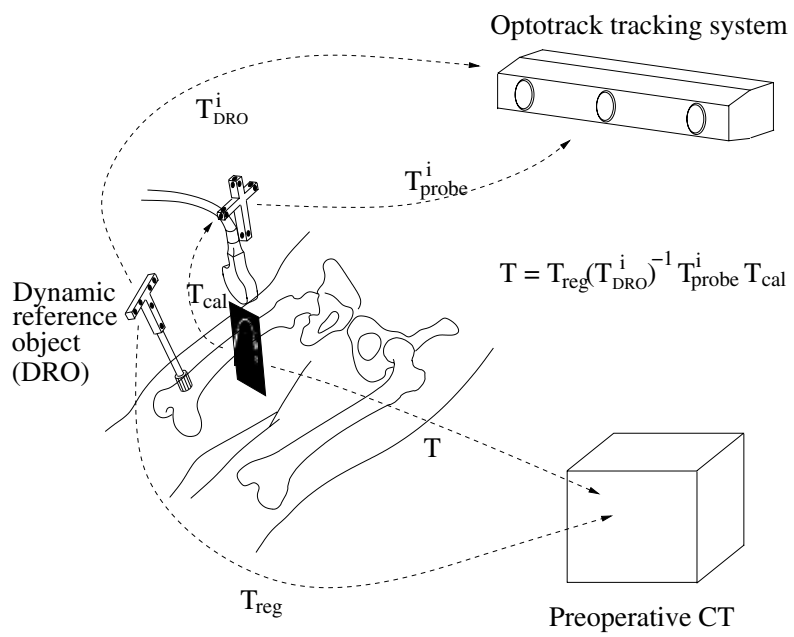

Fig. 1. Overview of system and transformations 
positions, $\mathbf{x}_{C T}$, within the CT volume. Transformation $\mathbf{T}$ is computed from four separate transformations:

$$
\mathbf{T}=\mathbf{T}_{r e g}\left(\mathbf{T}_{D R O}^{i}\right)^{-1} \mathbf{T}_{\text {probe }}^{i} \mathbf{T}_{\text {cal }}
$$

The calibration matrix, $\mathbf{T}_{\text {cal }}$, which transforms positions from the US image to positions relative to the infrared-emitting diodes (IREDs) attached to the probe was calculated using an invariant point method. Transformations $\mathbf{T}_{D R O}$ and $\mathbf{T}_{\text {probe }}$ transform positions relative to the IREDs (on the DRO and attached to the US probe respectively) to positions relative to the cameras on the Optotrak localiser.

The algorithm described in this paper calculates the transformation $\mathbf{T}$ by altering the six rigid-body parameters which define transformation $\mathbf{T}_{r e g}$ and the probe calibration scaling parameter in the vertical (or $y$ ) US image direction, $s_{y}$, in order to optimise the value of a similarity measure between the US and CT images. The scaling parameter in the horizontal (or $x$ ) US direction, which is not affected by the speed of sound in the imaged medium, was held constant during optimisation. After optimisation $\mathbf{T}_{r e g}$ can be used to determine the position and orientation of any tracked and calibrated object (such as a surgical drill) in the CT scan, and so can relate the intraoperative positions of such instruments to a preoperative plan described in the CT image.

The similarity measure for the algorithm is calculated as follows: The current estimate of $\mathbf{T}_{r e g}$ and $s_{y}$ are used to reslice the CT probability image (described in section 2.2) in the plane of each US slice (N.B. therefore, there is no requirement to compound the US slices to produce a $3 \mathrm{D}$ volume). The pixel values in these reformatted slices and in the US probability images are then compared using the normalised cross-correlation similarity measure. A three stage hill-climbing optimisation approach is used: low-resolution, high-resolution and then highresolution which includes $s_{y}$ optimisation.

\subsection{Formation of Probabilistic Images}

The US and CT images are converted from intensity images, $I(\mathbf{x})$, into probabilistic images, $P(\mathbf{x})$, where $\mathbf{x}$ represents the image position and $P(\mathbf{x})$ represents the probability of a pixel or voxel containing a bone-to-soft-tissue interface. The probability images, $P(\mathbf{x})$, are calculated using a probability density function $(\mathrm{PDF}), p$, which, given a set of image features, $\mathbf{F}(\mathbf{x})$, returns an estimate of the probability that position $\mathbf{x}$ is a bone-edge, i.e. $P(\mathbf{x})=p(\mathbf{F}(\mathbf{x}))$.

Converting the CT Volume: The CT PDF, $p_{C T}$, was based on two image features, so $\mathbf{F}(\mathbf{x})_{C T}=\mathbf{F}\left(f_{C T 1}(\mathbf{x}), f_{C T 2}(\mathbf{x})\right)$. Both CT features were calculated in 2D by applying operators to each CT slice in turn. The first CT feature, $f_{C T 1}(\mathbf{x})$, was the intensity of a gradient image calculated using Sobel operators. The second feature, $f_{C T 2}(\mathbf{x})$, was set equal to the maximum value under a $3 \times 3$ mask centred on pixel $\mathbf{x}$.

A set of training data, comprised of $n$ CT volumes, was used to calculate $p_{C T}$. Our aim is to identify two sets of voxels in the training data: all of the 
voxels which lie on a bone-to-soft-tissue boundary, $S_{\text {edgeCT}}$; and all the voxels in the training data, $S_{C T}$. Therefore, $S_{\text {edgeCT }} \subset S_{C T}$, and the PDF is defined as

$p_{C T}(a, b)=\frac{\text { Number of voxels where } \mathbf{x} \in S_{\text {edgeCT }} \text { with } f_{C T 1}(\mathbf{x})=a \text { and } f_{C T 2}(\mathbf{x})=b}{\text { Number of voxels where } \mathbf{x} \in S_{C T} \text { with } f_{C T 1}(\mathbf{x})=a \text { and } f_{C T 2}(\mathbf{x})=b}$

Converting the US Slices: The first step is an artifact removal stage. Our method uses simple knowledge of the US image formation process; in particular, that most strong reflections and artifacts cause a loss of signal intensity along the direction of the beam. The algorithm begins at the bottom of each column of pixels in the US image, and moves upwards towards the transducer face. The image is labelled as artifact until a threshold value is reached, see Figure 2(b). A small region (3mm) immediately adjacent to the transducer face is also labelled as artifact to remove artifacts caused by imperfect acoustic coupling at the skin surface boundary. Image regions labelled as artifact were not used in any subsequent image processing.

Two image features, $\mathbf{F}(\mathbf{x})_{U S}=\mathbf{F}\left(f_{U S 1}(\mathbf{x}), f_{U S 2}(\mathbf{x})\right)$, were used for the US $\mathrm{PDF}, p_{U S}$. The first US feature, $f_{U S 1}(\mathbf{x})$, was the intensity of the US image $I_{U S}(\mathbf{x})$. The second US feature, $f_{U S 2}(\mathbf{x})$, was the number of pixels below $\mathbf{x}$ not labelled as artifact. Both of these features are able to highlight bone edges due to the large change in acoustic impedance between soft-tissue and bone. This results in high US intensity values at the boundary (detected by $f_{U S 1}(\mathbf{x})$ ) followed by a bone shadow region of very low US intensities (detected by $f_{U S 2}(\mathbf{x})$ ). Figure 2 shows an example of an US probability image.

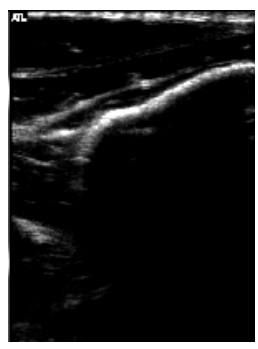

(a)

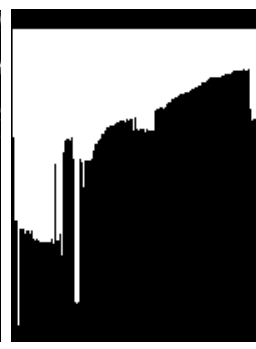

(b)

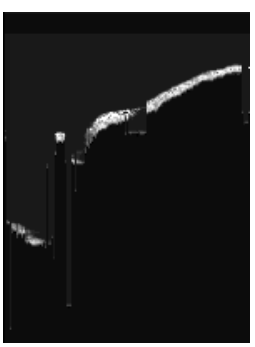

(c)

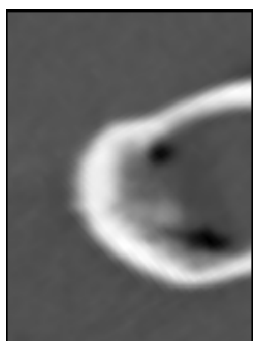

(d)

Fig. 2. Formation of US probability images: (a) sample US slice through femur; (b) mask produced by the artifact removal stage where only pixels in the white region are included for subsequent image processing; (c) probability image; (d) corresponding reformatted slice through the CT volume (calculated using the "gold standard" transformation) included to show the position of bone boundaries. In (c) Some nonbone-boundary pixels can be seen to have been allocated a high value in the probability image and vice-versa. However, it is important to note that our aim is not to produce a perfect segmentation, but to produce probability images of sufficient quality to allow accurate and robust registration. 
A set of US slices were used as training data for $p_{U S}$. The bone edge was defined in the training US images as a number of manually selected points connected by straight lines, to give the pixel set, $S_{\text {edgeUS }}$. The pixel set, $S_{U S}$, comprised all the non-artifact US pixels from the training data, and, as before, $S_{\text {edgeUS }} \subset S_{U S}$. The PDF, $p_{U S}$, is then defined as

$$
p_{U S}(a, b)=\frac{\text { Number of pixels where } \mathbf{x} \in S_{\text {edgeUS }} \text { with } f_{U S 1}(\mathbf{x})=a \text { and } f_{U S 2}(\mathbf{x})=b}{\text { Number of pixels where } \mathbf{x} \in S_{U S} \text { with } f_{U S 1}(\mathbf{x})=a \text { and } f_{U S 2}(\mathbf{x})=b}
$$

\section{$2.3 \quad$ Experiments}

Data Acquisition: for this study was carried out using 3 complete female cadavers. Fiducial markers were implanted into the femur and pelvis of each cadaver (4 in each femur and 5 in each hemi-pelvis) which were used to calculate a "gold standard" registration between image and physical space. A single high-resolution spiral CT scan (Siemens SOMATOM Plus 5) was obtained of the whole pelvis and femurs of each cadaver (voxel dimensions varied between $0.71 \times 0.71 \times 2 \mathrm{~mm}^{3}$ and $\left.0.79 \times 0.79 \times 2 \mathrm{~mm}^{3}\right)$. Each CT was then reduced into three smaller volumes which contained either a pelvis or femur plus some surrounding anatomy.

Prior to acquiring US images, a dynamic reference object (DRO) was rigidly attached to the bone. All measurements were recorded relative to the co-ordinate system of the DRO. The use of a DRO enabled the position of the cadaver to be changed during US acquisition so that images could be obtained in regions that would otherwise be inaccessible with the cadaver remaining in the supine position. The attachment of DROs [2,3] or external fixators [1] is standard practice for most image-guided orthopaedic surgery systems. Ultrasound images were acquired using a Philips-ATL HDI-5000 scanner and a high frequency probe (5-12 $\mathrm{MHz}$ ). Between 168 and 565 tracked US images were acquired of each bone using continuous scanning. This took approximately 8 minutes, though we believe that this time could be substantially reduced if an optimal protocol was established. The US images showed no effects of the cadaver preservation process, and so the acquired sets of US images should closely represent clinical data; both in terms of image characteristics, and in terms of in which regions of human femur and pelvis is it possible to obtain clear images of the bone surface using US.

Clinically Realistic Starting Positions: One method for obtaining a starting estimate for the algorithm in a clinical situation is to pick corresponding skin positions in the CT scan and physically using a tracked pointer. We have simulated the above process to obtain 100 starting estimates for each bone. The simulation uses four skin positions on each pelvis, and 3 skin positions and the pivot position for each femur. Our simulation assumes that the skin positions can be selected within a distance of $20 \mathrm{~mm}$ and the pivot position is accurate to within $10 \mathrm{~mm}$.

Training Data for PDF Calculations: For the experiments described in this paper all the CT femur images and between 42 and 100 US images from each femur were used as training data. In order to keep the training data separate from 
the experimental data the following method was used: All the pelvis experiments used the same US and CT PDFs, which were produced using all the training data. For the femur experiments, separate PDFs were produced for each femur. For a given femur, PDFs were produced using a subset of the training data. This subset comprised all the training data except for the data from the given femur.

\section{Results}

The results are shown visually using sample overlay images in Figure 3, and numerically in Table 1, where root mean square (RMS) target registration error (TRE) values have been calculated at three stages during the registration process: after the low-resolution optimisation, after the high-resolution optimisation and after the scaling parameter, $s_{y}$, is included in the optimisation. The

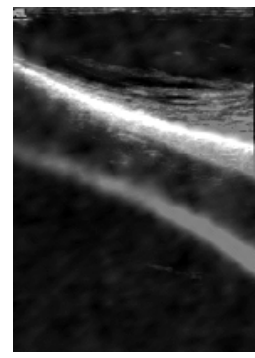

(a)

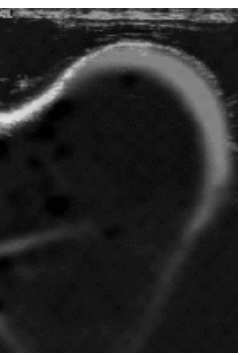

(b)

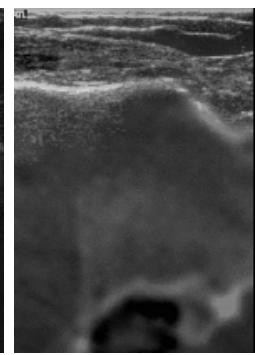

(c)

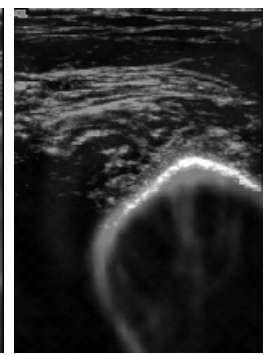

(d)

Fig. 3. Sample overlays of US onto reformatted slices through CT volumes calculated using the intensity-based registration transformation. Images correspond to (a) cadaver 1 pelvis, (b) cadaver 1 L.femur, (c) cadaver 2 L.femur and (d) cadaver 3 R.femur.

Table 1. Registration accuracy, mean RMS TRE, for each bone averaged over all successful registrations. The RMS TRE was calculated over the whole bone surface. A registration was defined to be a failure if its RMS TRE was more than double the mean RMS TRE calculated over all 100 registrations.

\begin{tabular}{|c|c|c|c|c|c|c|c|c|}
\hline \multirow[t]{2}{*}{$\mathrm{Cad}$} & \multirow[t]{2}{*}{ Bone } & \multicolumn{4}{|c|}{ mean (and s.d.) RMS TRE (mm) } & \multirow[t]{2}{*}{$s_{y}$} & \multirow{2}{*}{$\begin{array}{l}\text { No. } \\
\text { fail }\end{array}$} & \multirow{2}{*}{$\begin{array}{l}\text { No. US } \\
\text { slices }\end{array}$} \\
\hline & & initial & low-res & high-res 1 & inc. scaling & & & \\
\hline \multirow[t]{3}{*}{1} & L.Fem & $7.3(2.2)$ & $3.4(0.6)$ & $2.3(0.1)$ & $2.2(0.1)$ & 1.004 & 0 & 226 \\
\hline & R.Fem & $7.1(2.3)$ & $4.6(0.3)$ & $2.2(0.1)$ & $3.0(0.1)$ & 1.028 & 0 & 168 \\
\hline & Pelvis & $11.0(3.5)$ & $2.2(0.1)$ & $1.7(0.1)$ & $1.7(0.1)$ & 1.001 & 0 & 200 \\
\hline \multirow[t]{3}{*}{2} & L.Fem & $7.7(2.5)$ & $4.8(0.1)$ & $1.3(0.1)$ & $0.8(0.1)$ & 0.991 & 0 & 247 \\
\hline & R.Fem & $7.9(2.6)$ & $4.9(0.3)$ & $1.1(0.0)$ & $1.1(0.0)$ & 1.000 & 0 & 556 \\
\hline & Pelvis & $15.1(7.0)$ & $3.6(0.2)$ & $1.7(0.0)$ & $1.0(0.0)$ & 0.971 & 1 & 317 \\
\hline \multirow[t]{3}{*}{3} & L.Fem & $7.1(2.3)$ & $2.6(0.6)$ & $1.6(0.4)$ & $1.3(0.2)$ & 0.965 & 0 & 516 \\
\hline & R.Fem & $7.3(2.5)$ & $5.0(0.3)$ & $2.4(0.1)$ & $1.4(0.1)$ & 0.969 & 0 & 565 \\
\hline & Pelvis & $11.6(4.0)$ & $5.1(0.2)$ & $3.0(0.1)$ & $2.2(0.1)$ & 0.974 & 1 & 331 \\
\hline
\end{tabular}


factor by which $s_{y}$ has changed, the failure rate and the number of US slices used for each bone registration are also given. The results show that the mean RMS TRE after registration was less than $2.3 \mathrm{~mm}$ for all the registrations except for the right femur of cadaver 1 . The results for this case also show the only instance when the RMS TRE increased when the optimisation included the $y$ scaling parameter; when this parameter was held fixed, the RMS TRE was $2.3 \mathrm{~mm}$. These registrations used the smallest number of US images (only 168) compared to over 500 for some of the other femur registrations, and we believe that this may have been a factor in the higher TRE calculated from this case.

\section{Discussion and Conclusions}

Very basic image processing filters have been used to produce the features on which the probability images are calculated. These have proven sufficient to allow accurate and robust registrations. However, in the future other, more sophisticated filters may be used which are more accurate and robust in their ability to extract bone-to-soft-tissue edges from US and CT images.

The inclusion into the optimisation process of the US image scaling parameter, $s_{y}$, improved the TRE values for over half the registrations, once by more than $1 \mathrm{~mm}$. Only in one case did the error increase, and this is believed to be due to insufficient numbers of US images.

Future work includes establishing how many US images (and from which anatomical areas) are required for accurate registration. The algorithm speed needs to be improved: current time for registration is between 5 and 22 minutes on a $2.8 \mathrm{MHz}$ Intel Pentium 4 processor. We also intend to combine this intensitybased technique with a method which instantiates a shape model [14]. This links in well with the current trend in computer assisted orthopaedic surgery, which is towards the use of CT-free methods.

We have presented an automatic method to register freehand 3D US images to a CT volume of a pelvis or femur. Our method is based on a strategy in which we preprocess images to provide a probability map of corresponding features. This preprocessing step uses information gathered from a set of manually segmented training data. The algorithm also optimises the parameter which defines the speed-of-sound through tissue. Our method had been compared to a "gold-standard" registration based on bone implanted fiducial markers. Registrations have been validated using three cadavers ( 6 femurs and 3 pelves). Results show that our method is accurate to less than $2.3 \mathrm{~mm}$ (in all-but-one case), which should be sufficiently accurate for most total hip replacement procedures.

\section{Acknowledgment}

We would like to thank the EPSRC (grant number GR/R03525/01) for funding this project. Our thanks also go to Jens Krugman of BrainLab AG, Munich for his assistance in organising the experiments. 


\section{References}

1. Barger, W., Bauer, A., Borner, M.: Primary and revision total hip replacement using the ROBODOC ${ }^{r}$ system. Clinic. Orthopaedics and Related Res. (1998) 82-91

2. DiGioia, III, A., Jaramaz, B., Blackwell, M., Simon, D., Morgan, F., Moody, J., Nikou, C., Colgan, B., Aston, C., Labarca, R., Kischell, E., Kanade, T.: Image guided navigation system to measure intraoperatively acetabular implant alignment. Clinic. Orthopaedics and Related Res. (1998) 8-22

3. Nabeyama, R., Matsuda, S., Miura, H., Mawatarim, T., Kawano, T., Iwamoto, Y.: The accuracy of image-guided knee replacement based on computed tomography. J. Bone Joint Surg. (Br) 86-B (2004) 366-71

4. Berger, R.: Total hip arthroplasty using the minimally invasive two-incision approach. Clinic. Orthopaedics and Related Res. (2003) 232-241

5. DiGioia, III, A., Plakseychuk, A., Levison, T., Jaramaz, B.: Mini-incision technique for total hip arthroplasty with navigation. J. Arthroplasty 18 (2003) 123-128

6. Ault, T., Siegel, M.: Frameless patient registration using ultrasonic imaging. In: Proc. First International Symposium on Medical Robotics and Computer-assisted surgery. Volume 1. (1994) 74-82

7. Brendel, B., Winter, S., Rick, A., Stockheim, M., Ermert, H.: Registration of 3D CT and ultrasound datasets of the spine using bone structures. Comput. Aided Surg. 7 (2002) 146-155

8. Ionescu, G., Lavallée, S., Demongeot, J.: Automated registration of ultrasound with CT images: application to computer assisted prostate radiotherapy and orthopaedics. In Taylor, C., Colchester, A., eds.: Medical Imaging Computing and Computer-Assisted Intervention-MICCAI '99, Springer, Lecture notes in computer science 1679 (1999) 768-777

9. Amin, D., Kanade, T., DiGioia, III, A., Jaramaz, B.: Ultrasound registration of the bone surface for surgical navigation. Comput. Aided Surg. 8 (2003) 1-16

10. Tonetti, J., Carrat, L., Blendea, S., Merloz, P., Troccaz, J., Lavallée, S., Chirossel, J.: Clinical results of percutaneous pelvis surgery. Computer assisted surgery using ultrasound compared to standard fluoroscopy. Comput. Aided Surg. 6 (2001) 204211

11. Brendel, B., Winter, S., Rick, A., Stockheim, M., Ermert, H.: Bone registration with 3D CT and ultrasound data sets. In: Computer Assisted Radiology and Surgery 2003, Elsevier Sciences B.V. (2003) 426-432

12. Jaramaz, B., Nikou, C., Cavalier, B., Blendea, S., Labarca, R., DiGioia, III, A.: Experimental validation of ultrasound registration of long bones. In Langlotz, F., Davies, B., Bauer, A., eds.: 3rd Annual Meeting of the Int. Soc. for Computer Assisted Orthopaedic Surgery, Steinkopff Darmstadt (2003) 160-161

13. Penney, G., Blackall, J., Hamady, M., Sabharwal, Y., Adam, A., Hawkes, D.: Registration of freehand 3D ultrasound and magnetic resonance liver images. Med. Image Anal. 8 (2004) 81-91

14. Chan, C., Barratt, D., Edwards, P., Penney, G., Slomczykowski, M., Hawkes, D.: Cadaver validation of the use of ultrasound for 3D model instantiation of bony anatomy in image guided orthopaedic surgery. In: Medical Imaging Computing and Computer-Assisted Intervention-MICCAI '04, Springer (2004) 397-404 